RESEARCH ARTICLE

\title{
Enhanced intracellular availability and survival of hammerhead ribozymes increases target ablation in a cellular model of osteogenesis imperfecta
}

\author{
Y Smicun ${ }^{1}$, MW Kilpatrick ${ }^{1}$, J Florer ${ }^{2}$, I Toudjarska ${ }^{1}, \mathrm{G} \mathrm{Wu}^{3}$, RJ Wenstrup ${ }^{2}$ and $\mathrm{P}$ Tsipouras ${ }^{1}$ \\ ${ }^{1}$ Department of Pediatrics, University of Connecticut Health Center, Farmington, CT, USA; ${ }^{2}$ Cincinnati Children's Hospital Research \\ Foundation, Cincinnati, OH, USA; and ${ }^{3}$ Department of Medicine, University of Connecticut Health Center, Farmington, CT, USA
}

\begin{abstract}
Antisense hammerhead ribozymes have the capability to cleave complementary RNA in a sequence-dependent manner. In osteogenesis imperfecta, a genetic disorder of connective tissue, mutant collagen type I has been shown to participate in but not sustain formation of the triple helix. Selective ablation of mutant collagen gene transcript could potentially remove the mutant gene product and reverse the dominant-negative effect exerted by the abnormal protein. In earlier studies we showed that the hammerhead ribozyme Col1A1Rz547 selectively cleaved a mutant Col1A1 gene transcript in a murine calvarial osteobleast cell line. In order to test the possible therapeutic efficacy of this approach, a dramatic downregulation of the mutant transcript must be
\end{abstract}

achieved, a function directly related to high steady-state level of intracellular ribozyme. We report significantly enhanced expression of Col1A1Rz547 by vaccinia T7 polymerase following infection with an attenuated T7-pol vaccinia virus as shown both by the intracellular level of the ribozyme and the cleavage of the mutant Col1A1 gene transcript. We also describe the engineering of a multimeric ribozyme construct comprising eight subunits, which can self-cleave to monomers. These studies suggest the potential use of multimeric ribozymes expressed by a vaccinia-based system in the therapy of a variety of disorders.

Gene Therapy (2003) 10, 2005-2012. doi:10.1038/

sj.gt.3302108

Keywords: hammerhead ribozyme; osteogenesis imperfecta; collagen type I; vaccinia vector; multimeric ribozyme

\section{Introduction}

The application of nucleic acids as antisense reagents for the downregulation of expression of disease causing genes has been the focus of a great deal of attention for more than 20 years. ${ }^{1,2}$ Hammerhead ribozymes are small RNA molecules with a catalytic core of less than 40 nucleotides (nt) that can be resolved into a substrate and a catalytic strand..$^{3-7}$ It is possible that any RNA molecule can be cleaved by hammerhead ribozymes in trans, provided it contains a putative cleavage site. These ribozymes have been applied to downregulate a variety of genes, and their potential as therapeutic agents in the management of genetic disorders, particularly those whose pathogenesis is the result of a dominant-negative effect exerted by a mutant gene product, has been widely recognized. ${ }^{8-11}$

More recently, the discovery of the role of RNA interference (RNAi) in post-transcriptional gene silencing has led to the suggestion that a new class of RNA-based therapeutics might be developed. ${ }^{12-14}$ Unlike the antisense and RNAi approaches, which both rely on the action of the cellular machinery for their effect, hammerhead ribozymes act directly to cleave their target

Correspondence: P Tsipouras, Department of Pediatrics, University of Connecticut Health Center, 263 Farmington Avenue, Farmington, CT 06030, USA

Received 29 March 2003; accepted 30 May 2003
mRNA. Additionally, hammerhead ribozymes have the advantage of being able to discriminate between closely related target RNAs, even when the two RNA species differ by as little as a single nucleotide. ${ }^{15}$

Selective intracellular ablation is a strategy particularly suited to conditions like osteogenesis imperfecta (OI), a systemic disorder of connective tissue presenting with osseous fragility, short stature, hearing loss, dental defects, and blue sclerae. ${ }^{16,17}$ Mutations in either of the genes encoding the pro $\alpha 1(\mathrm{I})$ and pro $\alpha 2(\mathrm{I})$ chains of collagen type I have been identified in many affected individuals, and have been determined to be the underlying cause of OI. ${ }^{16}$

Several studies on the molecular pathology of OI have identified two mechanisms of collagen type I defects. ${ }^{16}$ In one, null mutations result in the synthesis of mutant collagen chains that cannot be incorporated in the collagen triple helix. This mechanism leads to the deposition of half normal levels of collagen type I in the extracellular matrix and is associated with a mild clinical phenotype. ${ }^{18}$ The second mechanism results from the incorporation of mutant protein into the collagen triple helix, thus disrupting the collagen fibrillar structure. These dominant-negative mutations are associated with phenotypes ranging in severity from moderate to lethal. ${ }^{16}$

Several murine models of OI have been described. ${ }^{19-22}$ Pereira $e a^{23}$ generated mice transgenic for an internally deleted human gene for the prox1(I) chain of collagen 
type I. The deleted minigene (pMG155) was designed to synthesize shortened pro $\alpha 1$ (I) chain which could participate in but not sustain the formation of the triple helix, thus depleting the endogenous procollagen type I through protein suicide. ${ }^{23,24}$ Wenstrup et $a l^{25}$ developed MC3T3-E1, a murine calvarial osteoblast cell line stably expressing pMG155 that has been used as a model system to study the effect of dominant-negative OI mutations.

We previously tested the use of an antisense ribozyme complementary to a particular sequence of the human mutant minigene target, pMG155, abundantly expressed in MC3T3-E1 cells. After intracellular delivery of a ribozyme expression construct, intracellular ribozyme transcript was detected along with a relative reduction of pMG155 mutant collagen transcript level. ${ }^{26}$ Thus, the ribozyme was shown to be biologically active and its potential value in the therapy of OI was demonstrated. However, the utility of this monomeric ribozyme was limited by the restrictions of the transient expression system used. Previous studies have established that even a small fraction of mutant protein is sufficient to produce a clinically severe OI phenotype. ${ }^{19}$ Therefore, in order to achieve a therapeutic effect, a dramatic downregulation of the mutant transcript might be necessary. This could be accomplished by increasing the level of ribozyme available intracellularly. We report here on two alternate yet complementary modalities aimed at achieving higher intracellular ribozyme availability: firstly, a multimeric ribozyme construct that produces a single transcriptional unit containing multiple copies of the ribozyme motif and capable of self-cleavage; ${ }^{27}$ secondly, infection with a modified vaccinia virus Ankara (MVA) that produces large quantities of recombinant T7 polymerase, which acts on T7 promoter bearing ribozyme sequence to achieve rapidly a high level of ribozyme transcript. ${ }^{28}$

\section{Results}

The octameric ribozyme cleaves both in cis and trans In vitro transcription of the octameric transcript demonstrated that the transcript efficiently underwent ciscleavage to produce the monomeric subunit.

Transcription was carried out in the presence of $8 \mathrm{mM}$ $\mathrm{MgCl}_{2}$ to minimize cis-cleavage during the transcription reaction, and synthesized transcript was subsequently incubated in $20 \mathrm{mM} \mathrm{MgCl}_{2}$ to assay for cis-cleavage activity. A ladder of fragments of between 70 and $\sim 500 \mathrm{nt}$, the sizes expected if the octameric transcript were to cis-cleave (Figure 1b), was observed following in vitro transcription. Subsequent incubation in the presence of the higher $\mathrm{MgCl}_{2}$ concentration led to accumulation of the $70 \mathrm{nt}$ monomeric subunit (Figure 1c). Incubation at $50^{\circ} \mathrm{C}$ resulted in increased cleavage as compared to $37^{\circ} \mathrm{C}$, as has been observed previously for hammerhead ribozymes. ${ }^{8}$ In addition, the octameric ribozyme was able to cleave its target sequence in trans. Incubation of a $205 \mathrm{nt}$ minigene target transcript with octameric ribozyme transcript led to accumulation of increasing amounts of the 130 and 75 nt subfragments expected if the ribozyme cleaved its putative cleavage site over time (Figure 1d). a
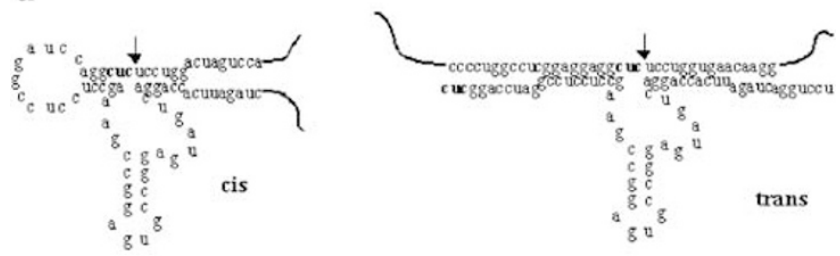

b

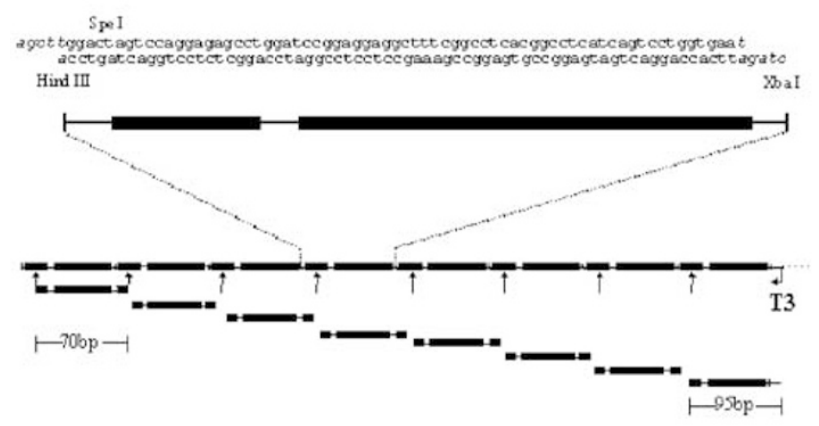

c

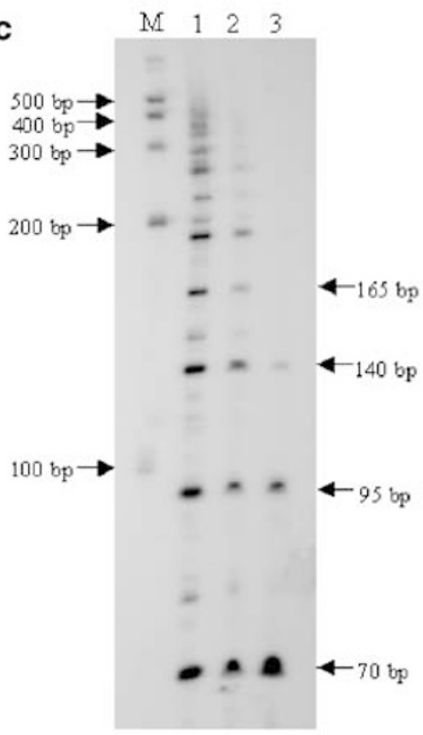

d

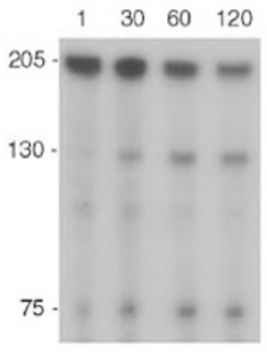

Figure 1 Structure and function of the octameric ribozyme designed to cleave both in cis and trans. (a) Monomeric Col1A1547Rz subunit designed to cleave both in cis and in trans. The ribozyme sequence is shown in the secondary structure that facilitates cis-cleavage on the left and is bound to its minigene target sequence on the right. The putative CUC cleavage site is shown in bold and the site of cleavage indicated by a vertical arrow. (b) Schematic representation of the octameric ribozyme construct. The sequence of the monomeric subunit is shown on top, with the octameric derivative underneath. Vertical arrows depict the expected sites of cis-cleavage of the octameric transcript. (c) In vitro transcription of the octameric ribozyme construct in the presence of $8 \mathrm{mM} \mathrm{MgCl}_{2}$ (lane 1) and incubation of the resultant transcript in $20 \mathrm{mM} \mathrm{MgCl}_{2}$ (lanes 2 and 3) at 37 or $50^{\circ} \mathrm{C}$, respectively. Lane $M$ contains an RNA marker ladder. Sizes of marker fragments are shown on the left and sizes of the fragments resulting from cis-cleavage of the transcript on the right. (d) In vitro ribozyme cleavage assay of the $205 \mathrm{nt}$ minigene target transcript with octameric ribozyme at $37^{\circ} \mathrm{C}$. Lanes $1,30,60$, and 120 show minigene target transcript incubated with ribozyme for 1, 30, 60, and $120 \mathrm{~min}$, respectively. The sizes of the full-length minigene transcript and the ribozyme cleavage products are shown on the left. 


\section{Vaccinia virus-mediated expression of ribozyme in cultured cells specifically downregulates the minigene target}

The MVA-T7 recombinant virus was used to achieve high level of ribozyme expression from the T7 promoter in minigene-expressing MC3T3-E1 cells. Cells were infected with crude virus stocks following transfection with plasmid constructs designed to express the Col1A1Rz547 ribozyme from the T7 promoter.

RNase protection analysis (RPA) of lysates from cells expressing the original monomeric Col1A1Rz547 ribozyme using the MVA-T7 system showed a substantial reduction in the amount of minigene transcript relative to control cyclophilin transcript compared to cells transfected with vector alone (Figure 2a). In addition, an $80 \mathrm{nt}$ protected subfragment (MGCF), the expected result of minigene cleavage by the ribozyme, appears only in cells expressing the monomeric ribozyme (Figure 2a).

Western blot analysis of cell lysates showed that the level of the truncated collagen encoded by the minigene is also reduced in MVA-T7-infected cells transfected with ribozyme construct compared with cells transfected with vector alone (Figure $2 b$ ). In contrast, the expression of the ribozyme did not affect the endogenous collagen protein.

Surprisingly, RPA of cells transfected with the octameric ribozyme construct showed little reduction in the amount of minigene transcript (Figure 2a).

\section{Efficacy of Col1A1Rz547 in cultured cells is dependent on ribozyme structure and survival}

The unexpected finding that the octameric ribozyme construct was less effective than the original monomeric Col1A1Rz547 construct when delivered to cultured cells led us to investigate the intracellular concentration of ribozyme produced by these constructs. RPA of lysates from cells transfected with monomeric or octameric ribozyme construct was performed using either monomeric or octameric ribozyme riboprobe (Figure 3a, b). Only a $43 \mathrm{nt}$ fragment is common to both the $\mathrm{Rz}$ monomer and the octameric ribozyme subunit (the cis/ trans R1 monomer). Thus the octameric transcript should protect a $43 \mathrm{nt}$ fragment of the $\mathrm{Rz}$ probe (Figure $3 \mathrm{c}-\mathrm{I}$ ), and vice versa. Comparison of this $43 \mathrm{nt}$ fragment demonstrated a much higher steady-state level of ribozyme in the monomeric Rz-expressing cells (Figure $3 b-R z)$ than in those expressing the octameric ribozyme (Figure 3a-R8).

The vector transcript would be expected to protect a $40 \mathrm{nt}$ fragment of the $\mathrm{Rz}$ probe; however, the $143 \mathrm{nt}$ protected fragment observed (Figure $3 a-V$ ) indicates that transcription proceeds around the plasmid in a 'rolling circle' manner (Figure 3c - II). Similarly, RNA from cells expressing the monomeric $\mathrm{Rz}$ (Figure $3 \mathrm{a}-\mathrm{Rz}$ ) protects both the predicted $182 \mathrm{nt}$ fragment and the fulllength $295 \mathrm{nt}$ probe fragment expected for a 'rolling circle' transcript (Figure 3c - III).

We hypothesize that the downstream vector sequence protects the monomeric ribozyme transcript from exonuclease degradation, while the octamer, following selfcleavage into the R1 subunits that lack any protective $3^{\prime}$ sequence, is rapidly degraded by RNases. Supporting
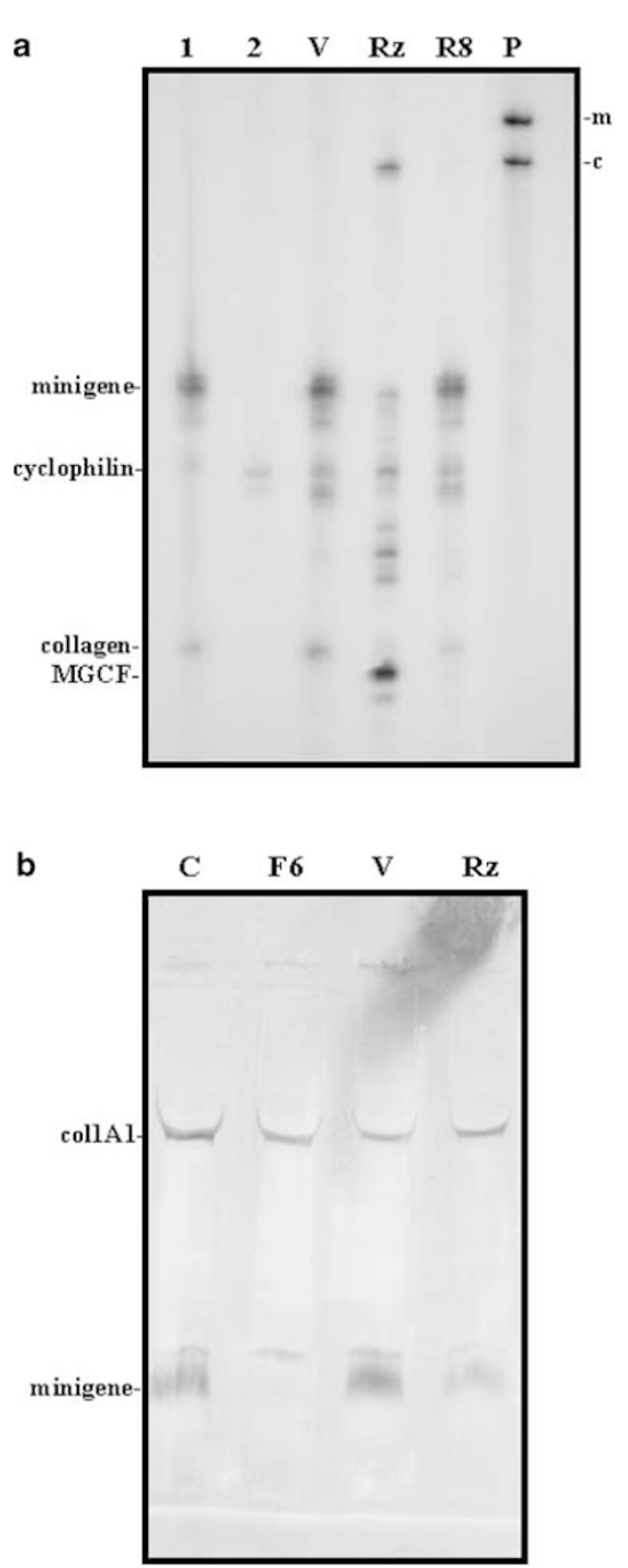

Figure 2 Effect of vaccinia virus-mediated expression of ribozyme in cultured cells on the levels of minigene target transcript and protein. (a) RPA of lysate from minigene-expressing cells transfected with ribozyme constructs. Lanes 1 and 2, RPA of cells transfected with vector alone using minigene or control cyclophilin riboprobe, respectively. Lanes $V, R z$, and R8, RPA of cells transfected with vector, monomeric ribozymes, or octameric ribozymes respectively, using both minigene and cyclophilin riboprobes. Lane P contains full-length minigene $(m)$ and cyclophilin (c) riboprobes. The position of the protected minigene and cyclophilin fragments, as well as endogenous collagen and the minigene cleavage fragment (MGCF), is shown on the left. The protected minigene fragment is detected as a doublet. (b) Western blot analysis of cell lysates using the LF41 antibody that recognizes both native murine col1A1 and the truncated collagen encoded by the minigene. Lane $C$, minigene-expressing cells. Lane F6, control cells that do not express the minigene. Lanes $V$ and $\mathrm{Rz}$, minigene-expressing cells transfected with vector or ribozyme construct, respectively. The position of mouse collA1 and the truncated collagen encoded by the minigene is indicated on the left.

this hypothesis is the decreasing intensity of the protected octamer probe by the cellular octamer RNA, as its size decreases (Figure $3 b-R 8$ ). 

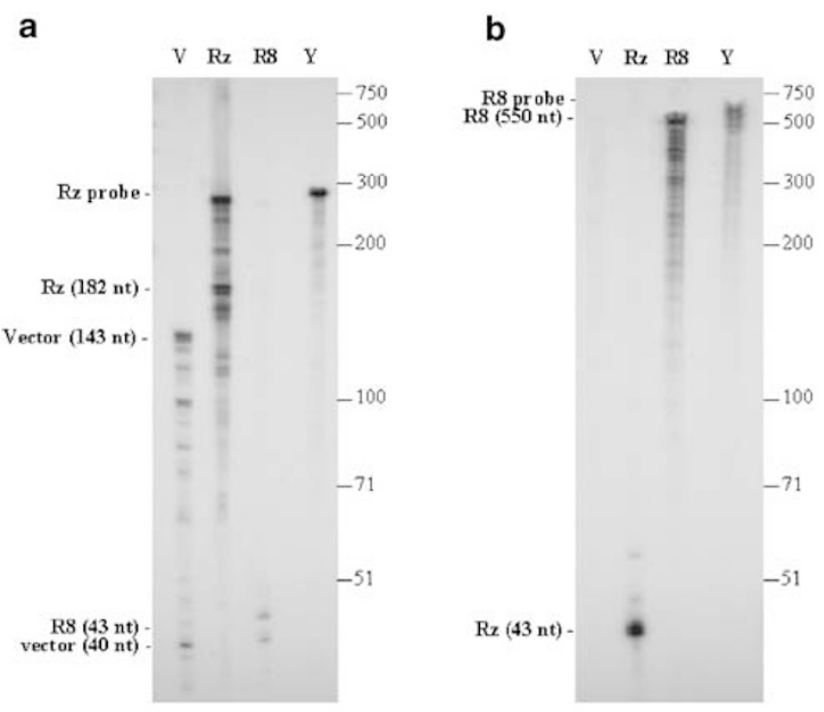

c

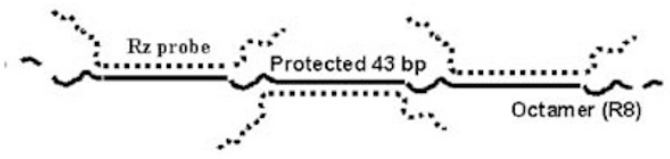

II

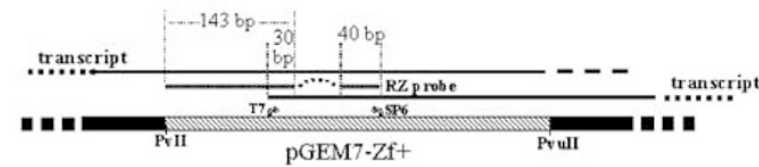

III

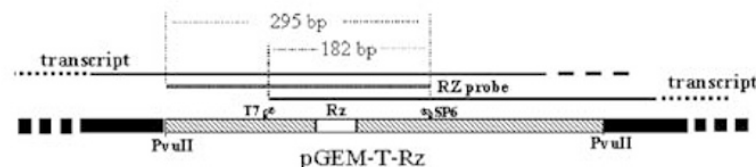

Figure 3 Efficacy of Col1A1Rz547 in cultured cells is dependent on intracellular ribozyme concentration. (a) RPA of lysate from minigeneexpressing cells transfected with monomeric (Rz) or octameric (R8) ribozyme constructs using a monomeric ribozyme riboprobe. Lane $V, R P A$ of cells transfected with vector alone. Lane $Y$, control RPA of yeast RNA without RNase treatment. Positions of the monomeric riboprobe ( $R z$ probe) and protected fragments, with sizes in parentheses, are shown on the left. Positions of size markers, in nucleotides, are shown on the right. (b) RPA of lysate from minigene-expressing cells transfected with monomeric $(R z)$ or octameric (R8) ribozyme constructs using an octameric ribozyme riboprobe. Lane $V, R P A$ of cells transfected with vector alone. Lane $Y$, control RPA of yeast RNA without RNase treatment. Positions of the octameric riboprobe ( $R 8$ probe) and protected fragments, with sizes in parentheses, are shown on the left. Positions of size markers, in nucleotides, are shown on the right. (c) Schematic representation depicting the origins of the protected fragments in panels $(a)$ and $(b)$. Cross-hybridization of the monomeric (Rz) probe and octameric (R8) ribozymes is shown at the top (I), the parental vector is shown in the middle (II), and the monomeric ribozyme (Rz) construct on the bottom (III).

This would predict that the level of intracellular ribozyme following transfection with a construct expressing the monomeric subunit of the octamer construct (R1) would be similar to that following transfection with the octameric construct. This level would be expected to be lower than that produced by transfection with the monomeric ribozyme $\mathrm{Rz}$ construct that lacks cis-cleavage activity. The level of the target minigene transcript would be expected to mirror the concentration of the intracellular ribozyme.

The data presented in Figure 4 demonstrate this to be the case. RPA of cells transfected with the original monomeric ribozyme construct showed a significantly higher level of intracellular ribozyme than that observed following transfection with either the cis/trans monomeric subunit or the octamer (Figure 4a, lanes Rz, R1, and $\mathrm{R} 8$, respectively). This is reflected in the level of minigene transcript detected (Figure $4 \mathrm{~b}$, lanes $\mathrm{Rz}, \mathrm{R} 1$, and R8) and the appearance of minigene cleavage product (Figure $4 \mathrm{~b}-\mathrm{MGCF}$ ).

The difference in efficacy between the monomeric ribozyme $\mathrm{Rz}$ and both the R1 subunit and octamer correlates with the length of $3^{\prime}$ sequence present. This length difference is due to the cis-cleaving activity incorporated into the latter two molecules. Given this difference, we decided to directly test the effect of the length of $3^{\prime}$ sequence on intracellular ribozyme concentration and efficacy. Transfection with a series of constructs designed to produce transcripts with different lengths of sequence $3^{\prime}$ to the ribozyme motif was investigated with respect to the subsequent concentration of both intracellular ribozyme and minigene transcript. The plasmid expressing the Col1A1Rz547 ribozyme was linearized at several sites for the production of transcripts that differ solely in the presence of vector-encoded sequence $3^{\prime}$ to the ribozyme motif that vary in length from 0 to 2150 bases in length. We also used the original circular plasmid construct that produces a ribozyme transcript with a $3^{\prime}$ extension longer than 3050 bases (see above). RPA of total RNA from cells transfected with these constructs, using minigene- and ribozyme-specific riboprobes, demonstrated that intracellular ribozyme survival and concomitant reduction in target minigene transcript were dependent on the length of $3^{\prime}$ vector transcript length (Figure 5).

\section{Discussion}

We have established the transient expression of hammerhead ribozymes in the murine calvarial osteoblast line

Figure 4 Intracellular level and consequent efficacy of Col1A1Rz547 in cultured cells is dependent on ribozyme transcript structure. (a) RPA of lysate from minigene-expressing cells transfected with vector alone $(V)$, the monomeric $(R z)$, the monomeric subunit $(R 1)$, or the octameric (R8) ribozyme constructs, using ribozyme and cyclophilin riboprobes. Lane 1, $R P A$ of cells transfected with the monomeric Rz using ribozyme riboprobe. Lane 2, RPA of cells transfected with vector alone using control cyclophilin riboprobe. Lanes 7 and 8, control RPA of yeast RNA with and without RNase treatment, respectively. The position of the protected ribozyme and cyclophilin fragments is shown on the left and the positions of the fulllength ribozyme ( $r$ ) and cyclophilin (c) riboprobes on the right. (b) RPA of lysate from minigene-expressing cells transfected with vector alone $(V)$, the original monomeric construct $(R z)$, the monomeric subunit (R1), or the octameric ribozyme construct (R8), using minigene and cyclophilin riboprobes. Lanes 1 and 2, RPA of cells transfected with vector alone using minigene or control cyclophilin riboprobe, respectively. Lanes 7 and 8, control RPA of yeast RNA with and without RNase treatment, respectively. The position of the protected minigene, cyclophilin, endogenous collagen, and the minigene cleavage (MGCF) fragments is shown on the left and the positions of the full-length minigene $(m)$ and cyclophilin (c) riboprobes are shown on the right. 
MC3T3-E1 as a model system to investigate their biological effect in downregulating the expression of the pMG155 minigene. We previously showed that the hammerhead ribozyme Col1A1Rz547, engineered to specifically cleave the sequence of the minigene target, was indeed able to cleave the target in vitro and in cellulo. ${ }^{26}$ However, in order to achieve substantive response, we needed to significantly increase the steadystate level of intracellular ribozyme.

The first approach was to synthesize a multimeric ribozyme, capable of self-cleaving into monomeric units, thus producing multiple ribozyme molecules per
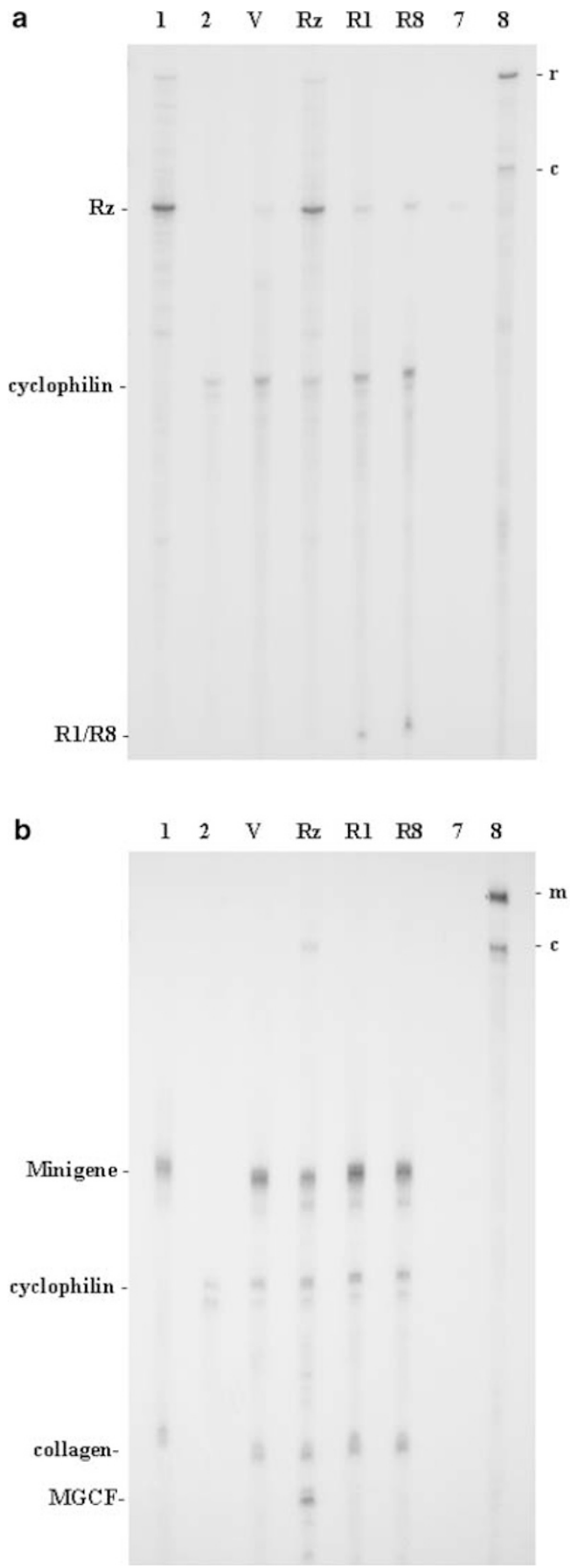

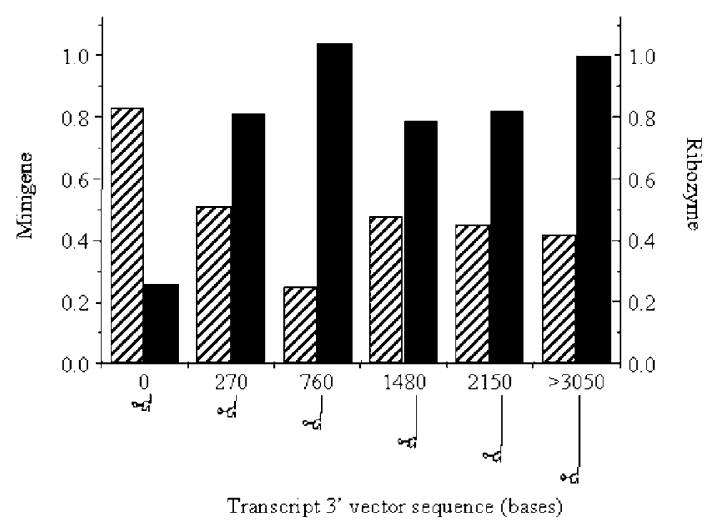

Figure 5 Effect of $3^{\prime}$ vector sequence on intracellular ribozyme concentration and efficacy. Quantitation of minigene and ribozyme transcripts following transfection with constructs that produce ribozyme transcripts containing from 0 to $>3050$ nt of $3^{\prime}$ vector sequence. Amount of minigene transcript (striped bar) is shown relative to transfection with vector alone (set to 1.0) and amount of ribozyme transcript (solid bar) relative to transfection with circular construct that produces a ribozyme transcript $>3050$ nt.

transcript. Transcription of a longer transcript is more efficient because reassembly of the transcriptional initiation machinery is a rate-limiting factor. The octameric ribozyme was shown to be capable of selfcleavage and of cleaving its intended target in the test tube.

The second approach was the use of the vaccinia/T7 polymerase system to express high levels of ribozyme. This polymerase is more efficient than eucaryotic RNA polymerases and can act in trans on $\mathrm{T} 7$ promoter bearing target sequences to achieve rapidly a high level of cytoplasmic gene expression. This system has been used successfully to express transiently high levels of a number of proteins and viral RNAs in cultured cells. Our data demonstrated significant decrease of mutant minigene transcript and of the corresponding mutant protein, consistent with previous observations following transfection with the trans-acting ribozymes. ${ }^{8,28}$ We decided to adopt this approach as a model system for the initial testing of ribozymes in cellulo to select those appropriate for stable expression.

We assumed that expression of the octamer by the vaccinia/T7 polymerase would offer an added advantage. To our surprise, expression of the octameric ribozyme did not reduce the level of the minigene target. RPA analysis revealed that the T7 polymerase continues transcription around the plasmid in a 'rolling circle' manner, as previously reported. ${ }^{29}$ We reasoned that the resultant downstream vector transcript sequence protects the ribozyme from cellular RNases, thus increasing its level in the cell. In contrast, the octamer ribozyme transcript undergoes cis-cleavage rendering the individual ribozyme motifs susceptible to RNase degradation. For this reason, the ribozyme level in cells transfected with the octamer was significantly lower compared to cells transfected with the original monomer. We assumed that for the same reason, the monomeric subunit would be equally susceptible to degradation, which proved to be true. Our hypothesis was further substantiated by the demonstration that both ribozyme level and consequent target cleavage were dependent on the length of downstream vector tail. 
Based on these data, it may be possible to define an optimal length and sequence of $3^{\prime}$ transcript for maximal cell survival and target cleavage. Furthermore, the insertion of a T7 termination signal into the appropriate position $3^{\prime}$ to the ribozyme motif might allow transcription of a ribozyme with optimal tail size from a circular plasmid. Alternative approach would be to utilize short sequences known to stabilize mRNAs. ${ }^{30}$

We have used the vaccinia/T7 polymerase system for transient expression studies. As the MVA strain does not usually replicate mammalian cells, we envision infection-transfection to be repeatable, thus prolonging ribozyme expression. Vaccinia virus has long been used for vaccination, and the mild reactions and side effects associated with its administration are well documented. ${ }^{31}$ This suggests that vaccinia could be used safely for gene therapy. ${ }^{31-33}$ Unlike retroviral vectors, vaccinia allows the transcription of genes with a polyadenylation signal, which improves transcript stability. ${ }^{34}$ An additional advantage is the cytoplasmic localization and lack of integration into the host genome. ${ }^{31,32,34}$

Large genomic deletions represent only a small fraction of OI mutations observed in the human, where the great majority are point mutations in a glycine codon. ${ }^{16}$ Glycine substitutions have been detected throughout the entire triple helical domain and have been associated with phenotypes ranging from mild to neonatal lethal. The lack of reduction of the endogenous murine collagen type I is in agreement with our earlier observations on the specificity of the ribozyme for its target. ${ }^{14}$ This feature could prove particularly useful in targeting sequences that are present in multiple copies as in the glycine residues of the triple helical domain of collagen type I.

\section{Materials and methods}

\section{Plasmid and virus preparation}

The construction of the minigene- and the Col1A1Rz547 ribozyme (Rz)-expressing vectors pGEM-T/547 and pGEM-T/Rz was previously described. ${ }^{26}$ The octameric ribozyme construct was synthesized by multimerization of a monomeric subunit comprising the ribozyme target sequence for cis-cleavage alongside the ribozyme sequence for trans-cleavage (Figure 1a). ${ }^{27}$ The cis-transmonomeric subunit (R1) flanked by HindIII and XbaI sticky ends was generated by annealing the two oligodeoxynucleotides shown in Figure $1 \mathrm{~b}$. The monomer was ligated into the HindIII and $\mathrm{XbaI}$ sites of pBluescript SK + (Stratagene, La Jolla, CA, USA) to yield the pBS-RZII plasmid. The monomeric subunit was excised from pBS-RZII using SpeI and XbaI enzymes, and ligated back into the vector in the reverse orientation to generate pBS-T7-Rzc, which was used to express the monomeric subunit.

The strategy used to produce tandem repeats of the monomeric subunit was based on the use of the restriction enzymes Xba I and Spe I that produce compatible sticky ends. Fragments produced by crossligation of $\mathrm{Xba}$ I-Spe I sticky ends are resistant to digestion by both enzymes, and thus successive ligation and digestion reactions produce fragments containing tandem repeats of the ribozyme fragment of known directionality. The monomeric subunit flanked by Xba I-Spe I restriction sites was self-ligated using T4 DNA ligase, a second Xba I-Spe I digestion performed, and the resulting fragments separated on an agarose gel. The band containing eight copies of the ribozyme was purified and ligated into Xba I-digested pBS547Rz to produce pBS-oct8.2 containing eight copies of the monomeric subunit sequence) (Figure 1b). To prevent deletion of rearrangements of the multimers, clones were propagated in the SURE strain (Stratagene, La Jolla, CA, USA).

The octamer was excised from pBS-oct8.2 using NotI and HindIII, and ligated into pcDNA3.1/Zeo(-) to generate $\mathrm{pD} 3 \mathrm{Rz} 8 \mathrm{mer}$ for expression of the octamer in cultured cells. For expression of ribozymes with defined length of downstream vector sequence, pGEMT/Rz was digested with NotI, PvuII, ApaLI, AvaII, or SspI, for generation of ribozyme transcripts with $0,270,760$, 1480 , or 2200 bases of downstream vector sequence, respectively.

The modified vaccinia virus Ankara expressing recombinant bacteriophage T7 polymerase (MVA-T7) was grown in BHK-21 cells as previously described ${ }^{35}$ for preparation of crude stocks.

\section{Cell culture and infection-transfection of cells}

The mouse calvarial cells MC3T3-E1 were maintained as described. ${ }^{25}$ For RPA, cells were plated on $100 \mathrm{~mm}$ plates at a concentration of 1.5 million cells/plate $24 \mathrm{~h}$ prior to infection-transfection. For Western analysis (WA), cells were plated in $35 \mathrm{~mm}$ wells at a concentration of 250000 cells/well. Cells were washed with PBS and incubated for $1 \mathrm{~h}$ in $20 \mu \mathrm{l}$ (WA-5 $\mu \mathrm{l})$ of crude MVA-T7 $\left(2 \times 10^{8} \mathrm{CFU} / \mu \mathrm{l}\right)$ preparation in $3 \mathrm{ml}(\mathrm{WA}-0.8 \mathrm{ml})$ of OptiMEM medium (Gibco, Carlsbad, CA, USA). A volume of $30 \mu \mathrm{g}$ (WA-5 $\mu \mathrm{g}$ ) of plasmid and $60 \mu \mathrm{l}$ (WA$10 \mu \mathrm{l}$ ) Lipofectamine 2000 (Invitrogen, Carlsbad, CA, USA) in $3 \mathrm{ml}$ (WA- $0.8 \mathrm{ml}$ ) medium were added and cells incubated for an additional 4-6h (WA-13 h). For RPA, cells were washed with PBS, detached by trypsin-EDTA (Gibco, Carlsbad, CA, USA) treatment, collected in growth medium, centrifuged, and lysed in Lysis/ De-naturation Solution (Ambion, Austin, TX, USA) (15000 cells $/ \mu \mathrm{l})$. For WA, cells were lysed, dialyzed, and lyophilized as described. ${ }^{25}$

\section{In vitro transcription and ribozyme cleavage}

Antisense minigene riboprobe was transcribed from pGEM-T/547 following linearization by NcoI. Ribozyme probe was transcribed from pGEM-T/Rz following digestion with PvuII (see Figure 5b), and cyclophilin probe was transcribed from pTRI-Cyclophilin (Ambion, Austin, TX, USA). Transcription reactions were performed using $50 \mu \mathrm{Ci} \alpha^{3}{ }^{32} \mathrm{P}-\mathrm{UTP}(800 \mathrm{ci} / \mathrm{mmol}$ ) (PerkinElmer, Boston, MA, USA), ATP, CTP, GTP (MBI, Hanover, MD, USA), and SP6 RNA polymerase (Invitrogen, Carlsbad, CA, USA) following manufacturers' specifications. The octamer probe and the 205 base radiolabeled minigene target RNA were transcribed from a $765 \mathrm{bp}$ PCR fragment generated from pBSoct8.2 using M13LacZ forward and reverse primers (Perkin-Elmer, Boston, MA, USA) and pGEM-T/547 using T7 RNA polymerase (Invitrogen, Carlsbad, CA, USA). Probes were purified either by a $6 \%$ denaturing PAGE or by NucAway spin columns (Ambion, Austin, TX, USA). Large-scale in vitro 
transcription of octamer ribozyme was carried out as described. ${ }^{26}$ Ribozyme cleavage reactions were carried out in a total volume of $30 \mu \mathrm{l}$ in $50 \mathrm{mM}$ Tris- $\mathrm{HCl} \mathrm{pH} 7.5$ and $20 \mathrm{mM} \mathrm{MgCl}_{2}$ for $0-120 \mathrm{~min}$ and were terminated by addition of $1 \mu \mathrm{l}$ of $0.5 \mathrm{M}$ EDTA.

\section{RNase protection assays}

RPA was carried out using Direct Protect Lysate RPA kit (Ambion, Austin, TX, USA). Lysates of 300000 cells were hybridized with $200000 \mathrm{cpm}$ of the appropriate riboprobes for $2 \mathrm{~h}$. Hybridization was at $55^{\circ} \mathrm{C}$ for minigene riboprobe or $53^{\circ} \mathrm{C}$ for ribozyme riboprobes. Following hybridization, digestion with RNase cocktail was for $4 \mathrm{~h}$ at $37^{\circ} \mathrm{C}$. Protected fragments were analyzed on denaturing PAGE and quantified as described. ${ }^{26}$

\section{Western analysis}

The minigene protein and endogenous pro $\alpha 1$ (I) collagen were detected by WA essentially as described previously. ${ }^{25}$. Briefly, samples were dialyzed against $0.5 \mathrm{M}$ acetic acid and lyophilized. Proteins were then separated by electrophoresis on SDS-PAGE gels, and electroblotted onto polyvinyldifluoride membranes (Osmonics Inc., Minnetonka, MN, USA). The membranes were reacted with polyclonal antisera to a peptide sequence from the carboxyl-propeptide of human pro 1 1(I) sequence (LF41).

\section{Acknowledgements}

This work was supported in part by a grant from the Children's Brittle Bone Foundation. Modified vaccinia virus Ankara expressing bacteriophage T7 polymerase (MVA-T7) was generously provided by Dr Bernard Moss, NIAID-NIH.

\section{References}

1 Opalinska JB, Gewirtz AM. Nucleic-acid therapeutics: basic principles and recent applications. Nat Rev Drug Discov 2002; 1: 503-514.

2 Lebedeva I, Stein CA. Antisense oligonucleotides: promise and reality. Annu Rev Pharmacol Toxicol 2001; 41: 403-419.

3 Cech TR, Bass BL. Biological catalysis by RNA. Annu Rev Biochem 1986; 55: 599-629.

4 Uhlenbeck OC. A small catalytic oligoribonucleotide. Nature 1987; 328: 596-600.

5 Haseloff J, Gerlach WL. Simple RNA enzymes with new and highly specific endoribonuclease activities. Nature 1988; 334: 585-591.

6 Forster AC, Symons RH. Self-cleavage of plus and minus RNAs of a virusoid and a structural model for the active sites. Cell 1987; 49: 211-220.

7 Scott WG et al. Capturing the structure of a catalytic RNA intermediate: the hammerhead ribozyme. Science 1996; 274: 2065-2069.

8 Kilpatrick MW et al. Delivery of a hammerhead ribozyme specifically down-regulates the production of fibrillin-1 by cultured dermal fibroblasts. Hum Mol Genet 1996; 5: 1939-1944.

9 Lewin AS et al. Ribozyme rescue of photoreceptor cells in a transgenic rat model of autosomal dominant retinitis pigmentosa. Nat Med 1998; 4: 967-971.
10 Scherr M et al. Effective reversal of a transformed phenotype by retrovirus-mediated transfer of a ribozyme directed against mutant N-ras. Gene Therapy 1998; 5: 1227-1234.

11 Millington-Ward $\mathrm{S}$ et al. A mutation-independent therapeutic strategem for osteogenesis imperfecta. Antisense Nucleic Acid Drug Dev 1999; 9: 537-542.

12 Hammond SM, Caudy AA, Hannon GJ. Post-transcriptional gene silencing by double-stranded RNA. Nat Rev Genet 2001; 2: 110-119.

13 Elbashir SM et al. Duplexes of 21-nucleotide RNAs mediate RNA interference in cultured mammalian cells. Nature 2001; 411: 494-498.

14 Hutvagner G, Zamore PD. RNAi: nature abhors a double-strand. Curr Opin Genet Dev 2002; 12: 225-232.

15 Phylactou LA, Tsipouras P, Kilpatrick MW. Hammerhead ribozymes targeted to the FBN1 mRNA can discriminate a single base mismatch between ribozyme and target. Biochem Biophys Res Commun 1998; 249: 804-810.

16 Byers PH, Cole WG. Osteogenesis imperfecta. In: Royce PM, Steinmann B (eds). Connective Tissue and its Heritable Disorders, 2nd edn. Wiley-Liss: New York, 2002 pp 385-430.

17 Kielty CM, Grant ME. The collagen family: structure, assembly, and organization in the extracellular matrix. In: Royce PM, Steinmann B (eds). Connective Tissue and its Heritable Disorders, 2nd edn. Wiley-Liss: New York, 2002 pp 159-221.

18 Barsh GS, David KE, Byers PH. Type I osteogenesis imperfecta: a non functional allele for pro $\alpha 1$ (I) chains of type I procollagen. Proc Natl Acad Sci USA 1982; 79: 3838-3842.

19 Stacey A et al. Perinatal lethal osteogenesis imperfecta in transgenic mice bearing an engineered mutant pro-alpha 1(I) collagen gene. Nature 1988; 332: 131-136.

20 Bonadio $\mathrm{J}$ et al. Transgenic mouse model of the mild dominant form of osteogenesis imperfecta. Proc Natl Acad Sci USA 1990; 87: 7145-7149.

21 Chipman SD et al. Defective pro alpha 2(I) collagen synthesis in a recessive mutation in mice: a model of human osteogenesis imperfecta. Proc Natl Acad Sci USA 1993; 90: 1701-1705.

22 Forlino A et al. Use of the Cre/lox recombination system to develop a non-lethal knock-in murine model for osteogenesis imperfecta with an alpha1(I) G349C substitution. Variability in phenotype in BrtlIV mice. J Biol Chem 1999; 274: 37923-37931.

23 Pereira $\mathrm{R}$ et al. Transgenic mice expressing a partially deleted gene for type I procollagen (COL1A1). A breeding line with a phenotype of spontaneous fractures and decreased bone collagen and mineral. J Clin Invest 1993; 91: 709-716.

24 Pereira $\mathrm{R}$ et al. Phenotypic variability and incomplete penetrance of spontaneous fractures in an inbred strain of transgenic mice expressing a mutated collagen gene (COL1A1). J Clin Invest 1994; 93: $1765-1769$.

25 Wenstrup RJ, Fowlkes JL, Witte DP, Florer JB. Discordant expression of osteoblast markers in MC3T3-E1 cells that synthesize a high turnover matrix. J Biol Chem 1996; 271: 10271-10276.

26 Toudjarska I et al. Delivery of a hammerhead ribozyme specifically downregulates mutant type I collagen mRNA in a murine model of osteogenesis imperfecta. Antisense Nucleic Acid Drug Dev. 2001; 11: 341-346.

27 Ruiz J, Wu CH, Ito Y, Wu GY. Design and preparation of a multimeric self-cleaving hammerhead ribozyme. Biotechniques 1997; 22: 338-345.

28 Carroll MW, Moss B. Poxviruses as expression vectors. Curr Opin Biotech 1997; 8: 573-577.

29 Cheng L, Goldman E. Reversal of inhibition by the T7 concatemer junction sequence on expression from a downstream T7 promoter. Gene Expression 2001; 9: 257-264.

30 Guhaniyogi J, Brewer G. Regulation of mRNA stability in mammalian cells. Gene 2001; 265: 11-23. 
31 Mastrangelo MJ, Eisenlohr LC, Gomella L, Lattime EC. Poxvirus vectors: orphaned and underappreciated. J Clin Invest 2000; 105: 1031-1034.

32 Evans ME, Lesnaw JA. Infection control in gene therapy [comment]. Infect Control Hosp Epidemiol 1999; 20: 568-576.

33 Peplinski GR, Tsung K, Norton JA. Vaccinia virus for human gene therapy. Surg Oncol Clin N Am 1998; 7: 575-588.
34 Konetschny C, Holzer GW, Falkner FG. Retroviral vectors produced in the cytoplasmic vaccinia virus system transduce intron-containing genes. J Virol 2002; 76: 1236-1243.

35 Carroll MW, Moss B. Host range and cytopathogenicity of the highly attenuated MVA strain of vaccinia virus: propagation and generation of recombinant viruses in a nonhuman mammalian cell line. Virology 1997; 238: 198-211. 